\title{
Pulmonary complications of predominantly antibody immunodeficiencies in a tertiary lung center
}

\author{
SEYED ALIREZA MAHDAVIANI ${ }^{1}$, SEPIDEH DAROUGAR ${ }^{1, *}$, DAVOOD MANSOURI $^{2}$, \\ SABEREH TASHAYOIE-NEJAD ${ }^{1}$, MAHSHID MOVAHEDI ${ }^{1}$, KARIM RAHIMI AGHDAM ${ }^{1}$, \\ HOSSEINALI GHAFFARIPOUR ${ }^{1}$, NOOSHIN BAGHAIE ${ }^{1}$, MARYAM HASSANZAD $^{1}$, \\ ALIREZA ESLAMINEJAD $^{3}$, ATEFEH FAKHARIAN $^{3}$, GUITTI POURDOWLAT $^{3}$, \\ JALAL HESHMATNIA ${ }^{3}$, MEHRDAD BAKHSHAYESHKARAM ${ }^{1}$, \\ MOHAMMADREZA BOLOURSAZ ${ }^{1}$, PAYAM TABARSI ${ }^{2}$, SEYED KAREN HASHEMITARI ${ }^{1}$, \\ ALI AKBAR VELAYATI ${ }^{4}$
}

\footnotetext{
${ }^{1}$ Pediatric Respiratory Diseases Research Centre, National Research Institute of Tuberculosis and Lung Diseases (NRITLD), Shahid Beheshti University of Medical Sciences, Tehran, Iran

${ }^{2}$ Clinical Tuberculosis and Epidemiology Research Centre, National Research Institute of Tuberculosis and Lung Diseases (NRITLD), Shahid Beheshti University of Medical Sciences, Tehran, Iran

${ }^{3}$ Chronic Respiratory Diseases Research Centre, National Research Institute of Tuberculosis and Lung Diseases (NRITLD), Shahid Beheshti University of Medical Sciences, Tehran, Iran

${ }^{4}$ Mycobacteriology Research Centre (MRC), National Research Institute of Tuberculosis and Lung Diseases (NRITLD), Shahid Beheshti University of Medical Sciences, Tehran, Iran

*Corresponding author: Dr. Sepideh Darougar; Pediatric Respiratory Diseases Research Centre, National Research Institute of Tuberculosis and Lung Diseases (NRITLD), Shahid Beheshti University of Medical Sciences, 19589-44413, P.O. Box 19575-154, Daar-Abad, Niavaran, Tehran, Iran; Phone: +98 91228819 75; Fax: +98 21261095 49; E-mail: sepidehdarougar@yahoo.com
}

(Received: May 23, 2018; Revised manuscript received: August 31, 2018; Accepted: September 20, 2018)

\begin{abstract}
Background and aims: Respiratory infections are expressed very soon in the life in humoral immunodeficiencies and often lead to chronic irreversible complications such as bronchiectasis and chronic airflow limitation. This study was conducted to evaluate the pulmonary complications of predominantly antibody immunodeficiencies to show the benefits of timely diagnosis and appropriate therapy. Patients and methods: The information of 48 patients involved with a type of predominantly antibody immunodeficiencies, including sex, type of primary immunodeficiency, age at the onset of symptoms, age at diagnosis, recurrent infections, respiratory symptoms, and pulmonary radiological and functional abnormalities were recorded and analyzed. Results: In 48 patients evaluated, the mean age at diagnosis was 25.63 years. The mean diagnostic delay was estimated to be 13.62 years. The most recurring clinical manifestations, sinusitis (69.6\%), otitis (43.5\%), and recurrent pneumonia were the cause of frequent admissions in $68.8 \%$ of these patients. Bronchiectasis was frequently found $(58.3 \%)$ in these patients mostly involving the middle and lower lobes (48.8\% and $41.5 \%$, respectively). Conclusions: Respiratory complications, infectious or non-infectious, determine the prognosis of the disease in patients with predominantly antibody immunodeficiencies. Timely diagnosis and appropriate management may improve life expectancy and the quality of life in these patients.
\end{abstract}

Keywords: primary humoral immunodeficiencies, complications, respiratory, morbidity, antibody

\section{Introduction}

Primary immunodeficiencies (PIDs) represent a growing group of chronic and serious conditions with profound effects on the quality of life and survival of the patients. Humoral immunodeficiencies, characterized by defective B cells and impaired antibody production, comprise about $70 \%$ of all PIDs with respiratory disease as a

This is an open-access article distributed under the terms of the Creative Commons Attribution-NonCommercial 4.0 International License, which permits unrestricted use, distribution, and reproduction in any medium for non-commercial purposes, provided the original author and source are credited, a link to the CC License is provided, and changes - if any - are indicated. 
significant cause of morbidity and mortality among affected patients $[1,2]$. More than 20 antibody deficiency diseases have been identified to date. However, many of them remain undefined [3]. As the first manifestations, respiratory infections are expressed very soon in the early life, usually within the first 5 years of age [4]. Recurrent pulmonary infections may often lead to chronic irreversible complications, such as bronchiectasis and chronic airflow limitation. However, these noninfectious complications are expected to appear later in the course of the disease in adolescent or adult life. Therefore, performance of careful and proper screening work-up for lung complications in patients with antibody deficiencies is crucial.

This study was conducted to evaluate the pulmonary complications of predominantly antibody immunodeficiencies in all age groups to show that timely diagnosis and appropriate therapy may decelerate the progression of these complications.

\section{Patients and Methods}

\section{Patients}

Forty-eight patients who fulfilled the inclusion criteria for predominantly humoral immunodeficiencies were enrolled in this database study. The follow-up of these patients was under a teamwork supervision of physicians fluent in management of lung disorders and also PIDs. The patients have been under close observation and monitoring since 2009 till data locking on 2017. Data were entered by treating physicians. Therefore, we believe that the registry approach provides valuable insight. All the patients were involved with a type of predominantly antibody immunodeficiency, including common variable immunodeficiency (CVID), X-linked agammaglobulinemia (XLA), and hyper IgM (HIGM) syndrome.

\section{Definitions}

CVID: CVID was referred to male or female patients with a marked decrease of IgG (at least 2 SD below the mean for age) and a marked decrease in at least one of the isotypes $\operatorname{IgM}$ or IgA [5] with an onset of immunodeficiency at greater than 2 years of age and absence of isohemagglutinins and/or poor response to vaccines after exclusion of cellular defects.

XLA: The diagnostic criteria for XLA in this study were male gender with less than $1 \%-2 \%$ of $\mathrm{CD}^{+} 9^{+}$ $B$ cells and mutation in Btk or absence of Btk mRNA on northern blot analysis of neutrophils or monocytes [6].
HIGM: It was characterized by recurrent infections, decreased serum levels of immunoglobulin G ( $\mathrm{IgG})$ and immunoglobulin $\mathrm{A}(\operatorname{IgA})$, and normal/increased serum levels of $\operatorname{IgM}[7,8]$.

Recurrent pneumonia: In this study, "recurrent pneumonia" was defined as "two or more episodes in 1 year or three or more episodes ever with complete radiographic resolution between episodes" [9].

\section{Study design}

This database study is a retrospective chart review of the patients referred to the Immunology Clinic of Dr. Masih Daneshvari Hospital with a type of predominantly antibody immunodeficiency. The patients were evaluated for their clinical features and basic immunological work-up. In the analysis of the clinical features, the following information including sex, type of PID, age at the onset of symptoms, age at diagnosis, recurrent infections, respiratory symptoms, and pulmonary radiological and functional abnormalities was considered and recorded.

The basic immunological work-up included complete blood count, serum immunoglobulin and complement levels, antibody response to vaccines, flow cytometric evaluation for the analysis of lymphocyte subsets, and nitroblue tetrazolium test.

The collected data were updated before the analysis.

\section{Ethics}

The study was approved by the ethics committee of Dr. Masih Daneshvari Hospital, Shahid Beheshti University of Medical Sciences, Tehran, Iran. An informed consent was obtained from all the patients.

\section{Statistical analysis}

Data analysis was performed using IBM SPSS Statistics for Windows, version 19 (IBM Corp., Armonk, NY, USA). Recognition of normal data was performed by the test of normality and Kolmogorov-Smirnov test. Quantitative data were described by mean statistics and standard deviation. Qualitative data and their count and percentage were presented by bar charts. T-test was used to compare the mean between two independent groups of data.

\section{Results}

This study was conducted on 48 patients, aged 3-54 years with primary humoral immunodeficiencies. 
Of all the patients evaluated, 27 (56.3\%) were male and $21(43.7 \%)$ were female with a male to female ratio of $1: 2$. The number of patients with CVID, XLA, and HIGM syndrome in this study was 42,2 , and 4 , respectively. Six patients from three unrelated pedigrees were family members: four patients from two of these pedigrees were siblings (two brothers with XLA and a brother and a sister with HIGM syndrome) and two patients with CVID were mother and daughter. Twelve $(25 \%)$ of the patients were in the pediatric age group (less than 18 years old) and $36(75 \%)$ were adults. The mean age at the onset of symptoms was 11.73 years with a mean age at diagnosis of 25.63 years. The mean diagnostic delay was estimated to be 13.62 years. Table I demonstrates the differences between the age at onset, diagnostic delay, and serum immunoglobulin levels according to specific immunodeficiencies studied.

The mean baseline serum IgG levels of all the patients studied were more than 2 SD below the normal level for all ages. The serum level of $\mathrm{IgA}$ was below $10 \mathrm{mg} / \mathrm{dl}$ in $16(33 \%)$ patients, of whom $10(27.1 \%)$ were adults and 6 (16.2\%) were children. The serum IgM level was lower than normal for age in only $3(8.1 \%)$ adults and $1(2.7 \%)$ child with primary humoral immunodeficiencies. The mean CD3 $\mathrm{T}$ cells were $76 \%$ with a range between $23 \%$ and $93 \%$. The mean CD4 T cells were $32 \%$ with a range between $11 \%$ and $72 \%$.

Among the events observed in these patients, recurrent respiratory symptoms, particularly of the upper respiratory tract, were the most frequent clinical manifestations (sinusitis: $69.6 \%$ and otitis: $43.5 \%$ ). Recurrent pneumonia was the cause of frequent admissions in $68.8 \%$ of these patients. Table II illustrates a comparison of certain respiratory manifestations in patients according to their specific immunodeficiencies.

To provide a documented determination of the pulmonary complications, a chest CT scan performed for the patients revealed abnormal findings in 40 (83.3\%) patients. Notably, bronchiectasis was particularly frequent (58.3\%) among our patients. It was predominantly bilateral and mostly involving the middle $(48.8 \%)$ and lower lobes $(41.5 \%)$. Upper lobe was involved only in $9.8 \%$ of cases with bronchiectasis (Fig. 1). The age group of patients with bronchiectasis was older regarding both during onset and diagnosis, although a significant difference was not detected. There was also no meaningful correlation between bronchiectasis and obstructive disease $(p$ value $=0.06)$ or restrictive disease $(p$ value $=0.2)$ either.

The most frequent finding on the thoracic CT other than bronchiectasis was bronchial wall thickening, which was detected in 11 (22.9\%) patients. There was no significant relationship between this finding with neither obstructive $(p$ value $=0.09)$ nor restrictive $(p$ value $=0.6)$ patterns of airway dysfunction. Other abnormal patterns in imaging of these patients were ground-glass opacities, mosaic attenuation, mediastinal lymphadenopathy, fibrosis, and parenchymal nodules.

Table I Demonstration of diagnostic delay and serum immunoglobulin levels in patients with X-linked agammaglobulinemia (XLA), hyper IgM (HIGM), and common variable immunodeficiency (CVID)

\begin{tabular}{lccc} 
& XLA (mean) & HIGM (mean) & CVID (mean) \\
Age at onset (years) & 2.75 & 1.75 & 13.07 \\
Age at diagnosis (years) & 6.5 & 11.67 & 27.93 \\
Diagnostic delay (years) & 3.75 & 9.92 & 14.56 \\
Serum IgG level $(\mathrm{mg} / 100)$ & 340 & 366 & 252.4 \\
Serum IgM level $(\mathrm{mg} / 100)$ & 40 & 1,107 & 41.4 \\
Serum IgA level $(\mathrm{mg} / 100)$ & 20 & 13.3 & 25.5 \\
\hline
\end{tabular}

Table II Common sites for respiratory involvement according to specific diagnoses

\begin{tabular}{lccc} 
Immunodeficiency & XLA: number of patients (\%) & HIGM: number of patients (\%) & CVID: number of patients (\%) \\
Recurrent pneumonia & $2(100 \%)$ & $3(75 \%)$ & $28(66 \%)$ \\
Sinusitis & $2(100 \%)$ & $2(50 \%)$ & $28(66 \%)$ \\
Otitis & $1(50 \%)$ & $3(75 \%)$ & $16(38 \%)$ \\
Abnormal CT & $2(100 \%)$ & $3(75 \%)$ & $35(83 \%)$ \\
Total number of patients & 2 & 4 & 42 \\
\hline
\end{tabular}

XLA: X-linked agammaglobulinemia; HIGM: hyper IgM; CVID: common variable immunodeficiency 


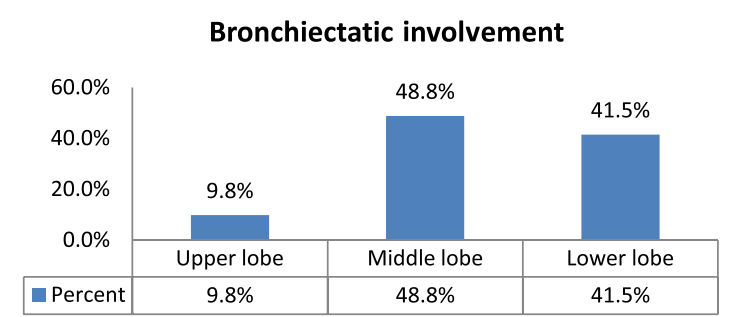

Fig. 1. An illustration of the distribution of bronchiectasis in different lobes of the lungs

Mosaic attenuation pattern was detected in highresolution computed tomography (HRCT) scan in $12 \%$ of the patients in this study.

Pulmonary function tests (PFTs) were performed for every patient who could complete the test procedure properly. Twelve $(37.5 \%)$ had normal PFTs, while spirometry revealed obstructive pulmonary defect in 10 $(31.3 \%)$ and restrictive pulmonary defect in $8(25 \%)$ patients. Two $(6.7 \%)$ of the patients showed a mixed pattern of restrictive and obstructive diseases in their PFT records. FEVI and FVC were less than $80 \%$ predicted in $78.9 \%$ and $76.3 \%$ of the patients, respectively. There was no meaningful relationship between age and the development of obstructive $(p$ value $=1)$ or restrictive diseases $(p$ value $=0.07)$ in this study.

We also found that $82.1 \%$ of patients with recurrent pneumonias had FVC $<80 \%$ predicted and $63.2 \%$ had FEVI $<80 \%$ predicted between their infectious episodes even when they were free of infectious lung involvements. Recurrent pneumonias did not have significant relation with abnormal CT scan findings in the patients studied $(p$ value $=0.2)$.

Among the immune-deficient patients with bronchiectasis, FEVI $<80 \%$ predicted and FVC $<80 \%$ predicted were detected in $55.3 \%$ and $52.6 \%$ of patients, respectively. We also found a significant relationship between bronchiectasis in the CT scans of the patients with obstructive disease $(p$ value $=0.03)$ but not with restrictive disease $(p$ value $=0.08)$ in our patients.

\section{Discussion}

Humoral immunodeficiencies are clinically the most important and largest group of inherited immune defects [10] with respiratory infections as their most common presenting feature [11]. The focus of this study is on respiratory manifestations of patients with predominantly antibody immunodeficiencies.

In several studies, PIDs show a male preponderance in patients particularly in pediatric age group [12-15]. Whereas in adult patients, a female preponderance $(56.3 \%)$ had been detected in a study conducted in Switzerland [15], which was referred to the prevalence of CVID as the most common PID. In this study, despite the fact that the patients evaluated were only those with predominantly antibody immunodeficiencies, we again found a male:female ratio of 1.2:1, a trend that has also been found in other studies when evaluating all types of PIDs.

Humoral immune deficiencies have a variable age at onset with an extended range from early childhood to adult life. Diagnostic delay, although inevitable, is an important contributor to morbidity and adversely affects the outcomes [16]. In a study published in Iran in 2011 [17], a diagnostic delay ranging from 2.5 to 5 years in children and adults was reported.

In this study, in adult patients, the median age at onset of signs and symptoms was 15.5 years and there was a mean diagnostic delay of 16 years, which indicates insufficient recognition of immunodeficiency disorders by primary care physicians in the past. In addition, the respiratory nature of these disorders made the immunologic basis of the disease to remain undiagnosed until they developed lung complications and were referred to a tertiary center. Underdiagnosis, diagnostic delays, and incorrect managements by various medical specialists were the main contributory factors, which contributed in developing morbidities before performing a proper diagnosis in those patients in this study. However, the average of age at onset and diagnosis among the children in this study was 4.3 and 8.4 years, respectively, representing a mean diagnostic delay of about 4 years in children. This is a reasonable period of time, consistent with published literature from advanced centers. We attribute this shortened period of delay in diagnosis in pediatric age group compared to adults to accelerated and significant progress in the field of immunodeficiency and also improved analytical techniques nowadays.

Respiratory infections including otitis media and sinusitis with an estimated frequency of more than $50 \%$ of cases are the most common presenting features, which make these patients seek medical help $[12,18$, 19]. In this study, acute otitis media (43.5\%) and sinusitis $(69.6 \%)$ were not only common findings among the adult patients but also in children, detected in $50 \%$ and $58.3 \%$ of them, respectively. The patients were also reported to experience lower respiratory tract infections and their complications including pneumonia (58.5\%) with a significant reduction in the number of sinopulmonary infections after initiation of replacement therapy in these patients.

Although $\mathrm{CD}^{+} \mathrm{T}$ cell levels adjusted by the age of the patients were lower than normal in $22(45 \%)$ patients in this series, their absolute counts were enough to prevent the development of opportunistic infections. None of these patients experienced such an infection in the course of their disease to date.

Chest CT scan plays a crucial role in detection of pulmonary changes, which are mainly the result of 
repeated and prolonged infections [20]. Bierry et al. reported pulmonary changes in $60 \%$ of patients with humoral immunodeficiencies. Generally, the risk of chronic lung disease is higher in patients with CVID than those with XLA, probably due to a longer diagnostic delay in CVID patients [10]. This was not true in XLA patients in this study who were referred to this center very late and with advanced lung disease due to delay in diagnosis. Abnormal CT scan was detected in $83.3 \%$ of our patients. We explained this high rate of abnormal findings with the advanced pulmonary involvement of our patients during their presentation, which was relatively late in the course of the disease. However, abnormal chest imaging was also frequently found in children, as $91.7 \%$ of our patients in this age group revealed some degrees of abnormalities in their chest CT scans, which could not be referred to as an outcome of significant diagnostic delay in this age group and was predominantly the result of repeated and prolonged infections.

Small airway involvement is relatively common in CVID that leads to ventilation abnormalities and even chronic obstructive disease, which appears at CT imaging as areas of low attenuation. Twelve percent of the patients in this study had mosaic attenuation pattern in their chest CT scan.

Recurrent pulmonary infections may lead to irreversible complications found in CT scan, such as bronchiectasis, bronchial wall thickening, nodular changes, consolidation processes, emphysema, and fibrotic changes. Bronchiectasis has been reported as the most common pulmonary complication in patients with primary humoral immune deficiencies [12, 21]. Pu et al. [22] estimated that $7 \%$ of patients with bronchiectasis have humoral immune defects, which are unfortunately diagnosed late, in the irreversible phase [23]. The frequency of bronchial abnormalities including bronchiectasis and bronchial wall thickening is reported to be between $14 \%$ and $60 \%$, with their presence indicative of a poor prognosis suggestive of the evolution of the disease process to an irreversible stage of lung fibrosis [20]. Bronchiectasis was also the most common pulmonary complication in this study with a frequency of $53 \%$ in all the patients and $41.7 \%$ in pediatric age group. Bronchiectasis was cylindrical, bilateral, and diffuse, most commonly in the middle or lower lobes with mucoid impaction in several instances. We attribute the high prevalence of bronchiectasis in these patients to factors including recurrent infections and persistent inflammation due to low levels of serum immunoglobulins during the diagnosis and the delay in both diagnosis and treatment. Moreover, it was confirmed in this study that bronchiectasis in XLA is an earlier event compared to bronchiectasis in CVID, probably due to an earlier onset of pulmonary infections in the former. Both of the siblings with XLA in this study suffered from diffuse-generalized bronchiectasis (leading to lobectomy before teenage years in the older brother). We found bronchiectasis only in three children with diagnosis of CVID.

We detected bronchial wall thickening in another $26.8 \%$ of our patients still without development of bronchiectasis. In pediatric age group, only $7.5 \%$ had bronchial wall thickening. We interpreted this finding as an early indicator of the possible development of bronchiectasis in near future.

Interstitial lung disease (ILD) particularly in patients with CVID presents with air trapping, bronchial wall thickening, bronchiectasis, emphysema, reticular, nodular, or ground glass opacities and parenchymal consolidation [24] in HRCT scan.

It has been estimated that $20 \%$ of patients with humoral immunodeficiencies develop an ILD. These patients tend to be over the age of 40 years and to have a prior history of recurrent infections. However, only $6 \%$ of the patients in this study were suffering from ILD in the course of their disease, of whom, two were deceased due to the severity of lung involvement. One of these two had granulomatous lymphocytic ILD. We explained the lower prevalence of ILD in our patients with the mean age of the patients, which was approximately 26 years. This younger age of the patients might be regarded as a reasonable clue for the lower prevalence of ILD in this study.

Ventilation abnormalities, particularly chronic obstructive disease, are relatively common events in patients with humoral immunodeficiencies.

Data from this interim analysis illustrate that patients with bronchiectasis showed significantly lower FVC and FEVI values compared to patients without bronchiectasis. Of those patients with bronchiectasis, and who are able to perform respirometry, $55.6 \%$ had a FEVI less than $80 \%$ and $52.8 \%$ had a FVC less than $80 \%$. Obstructive airflow limitation even without bronchiectasis was also found in $12 \%$ of patients.

Chronic airflow obstruction was observed in the early phases of the disease, whereas restriction seems to occur in the advanced stages of the disease. However, we could not find a significant relationship between age and restrictive $(p$ value $=0.07)$ or obstructive $(p$ value $=1)$ lung diseases Our results regarding obstructive airway disease (in $31.3 \%$ of patients) as a common event in PIDs were consistent with previous studies with a reported frequency of $15 \%-42 \%$ in antibody deficiencies $[25,26]$. Restrictive changes may be caused due to lymphocytic or granulomatous infiltrates or mucous plugging, somehow occurring at more advanced phases of the disease. In this study, it was detected in $25 \%$ of patients. The only two siblings with XLA in this study showed restrictive pattern in their PFT, which was consistent with the results of previous studies [27]. 


\section{Conclusions}

The purpose of this study was to gain insight into how primary antibody deficiencies might affect patient's health status by focusing on diverse pulmonary complications, which significantly influence the patients' state of wellbeing, quality of life, and survival. Timely diagnosis and appropriate management may considerably improve life expectancy and the quality of life in these patients.

In fact, respiratory complications, infectious or noninfectious, determine the prognosis of the disease. Because of the complicated course of these disorders, regular examinations and close monitoring of this vulnerable population using multidisciplinary health care teams are strongly recommended.

$$
* * *
$$

Funding sources: No Financial support was received for this study.

Authors' contribution: SAM and SD: study design, acquisition of data, drafting of the manuscript, literature review, and critical revision of the manuscript. DM, ST-N, MM, KRA, HG, NB, MH, AE, AF, GP, JH, $\mathrm{MB}, \mathrm{MB}, \mathrm{PT}$, and $\mathrm{SKH}$ : acquisition of data, literature review, and critical revision of the manuscript. AAV: study supervision. All authors take responsibility for the integrity and the accuracy of the data.

Conflict of interest: None.

Acknowledgements: The authors would like to thank Dr. Amir Hashemi Tari, Consultant Psychiatrist at East London NHS Foundation Trust, London, United Kingdom. This article has been extracted from the thesis written by Dr. Mahshid Movahedi in School of Medicine, Shahid Beheshti University of Medical Sciences (registration number: 63M).

\section{References}

1. Hampson F, Chandra A, Screaton N, Condliffe A, Kumararatne D, Exley A, Babar JL: Respiratory disease in common variable immunodeficiency and other primary immunodeficiency disorders. Clin Radiol 67, 587-595 (2012)

2. Dosanjh A: Chronic pediatric pulmonary disease and primary humoral antibody based immune disease. Respir Med 105, 511-514 (2011)

3. McCusker C, Warrington R: Primary immunodeficiency. Allergy Asthma Clin Immunol 7, S11 (2011)

4. Nonas S: Pulmonary manifestations of primary immunodeficiency disorders. Immunol Allergy Clin 35, 753-766 (2015)

5. Cunningham-Rundles C, Warnatz K (2014): Hypogammaglobulinemia and common variable immunodeficiency. In: Stiehm's Immune Deficiencies 1, eds Sullivan KE, Stiehm ER, Elsevier, London, p. 347

6. Plebani A, Lougaris V (2014): Agammaglobulinemia. In: Stiehm's Immune Deficiencies 1, eds Sullivan KE, Stiehm ER, Elsevier, London, p. 330

7. Patiroglu T, Akar HH, van der Burg M, Unal E: Autosomal recessive hyper $\operatorname{IgM}$ syndrome associated with activation-induced cytidine deaminase gene in three Turkish siblings presented with tuberculosis lymphadenitis - Case report. Acta Microbiol Immunol Hung 62, 267-274 (2015)

8. Durandy A, Kracker S (2014): Class-switch recombination defects. In: Stiehm's Immune Deficiencies 1, eds Sullivan KE, Stiehm ER, Elsevier, London, p. 368
9. Hughes D: Recurrent pneumonia... Not! Paediatr Child Health $18,459-460$ (2013)

10. Jesenak M, Banovcin P, Jesenakova B, Babusikova E: Pulmonary manifestations of primary immunodeficiency disorders in children. Front Pediatr 2, 77 (2014)

11. Costa-Carvalho BT, Wandalsen GF, Pulici G, Aranda CS, Solé D: Pulmonary complications in patients with antibody deficiency. Allergol Immunopathol 39, 128-132 (2011)

12. Membrila-Mondragon J, Staines-Boone AT, Sanchez-Sanchez LM, Ruiz-Pedraza MD: Pulmonary complications in pediatric patients with primary immunodeficiency. Gac Med Mex 151, 157-163 (2015)

13. de Silva NR, Gunawardena S, Rathnayake D, Wickramasingha GD: Spectrum of primary immunodeficiency disorders in Sri Lanka. Allergy Asthma Clin Immunol 9, 50 (2013)

14. Noh LM, Nasuruddin B, Abdul Latiff A, Noah R, Kamarul Azahar M, Norzila M: Clinical-epidemiological pattern of primary immunodeficiencies in Malaysia 1987-2006: A 20 year experience in four Malaysian hospitals. Med J Malaysia 68, 13-17 (2013)

15. Marschall K, Hoernes $M$, Bitzenhofer-Grüber $M$, Jandus $P$, Duppenthaler A, Wuillemin W, Rischewski J, Boyman O, Heininger U, Hauser T, Steiner U, Posfay-Barbe K, Seebach J, Recher M, Hess C, Helbling A, Reichenbach J: The Swiss National Registry for Primary Immunodeficiencies: Report on the first 6 years' activity from 2008 to 2014. Clin Exp Immunol 182, 45-50 (2015)

16. Edgar J, Buckland M, Guzman D, Conlon N, Knerr V, Bangs C, Reiser V, Panahloo Z, Workman S, Slatter M, Gennery AR, Davies EG, Allwood Z, Arkwright PD, Helbert M, Longhurst HJ, Grigoriadou S, Devlin LA, Huissoon A, Krishna MT, Hackett S, Kumararatne DS, Condliffe AM, Baxendale H, Henderson K, Bethune C, Symons C, Wood P, Ford K, Patel S, Jain R, Jolles S, El-Shanawany T, Alachkar H, Herwadkar A, Sargur R, Shrimpton A, Hayman G, Abuzakouk M, Spickett G, Darroch CJ, Paulus S, Marshall SE, McDermott EM, Heath PT, Herriot R, Noorani S, Turner M, Khan S, Grimbacher B: The United Kingdom Primary Immune Deficiency (UKPID) Registry: Report of the first 4 years' activity 2008-2012. Clin Exp Immunol 175, 68-78 (2014)

17. Aghamohammadi A, Bahrami A, Mamishi S, Mohammadi B, Abolhassani H, Parvaneh N, Rezaei N: Impact of delayed diagnosis in children with primary antibody deficiencies. J Microbiol Immunol Infect 44, 229-234 (2011)

18. Ardeniz Ö, Basoglu OK, Günsar F, Ünsel M, Bayraktaroglu S, Mete $\mathrm{N}$, Gülbahar O, Sin A: Clinical and immunological analysis of 23 adult patients with common variable immunodeficiency. J investig Allergol Clin Immunol 20, 222 (2010)

19. Bonilla FA, Geha RS: Primary immunodeficiency diseases. J Allergy Clin Immunol 111, S571-S581 (2003)

20. Bierry G, Boileau J, Barnig C, Gasser B, Korganow AS, Buy X, Jeung MY, Roy C, Gangi A: Thoracic manifestations of primary humoral immunodeficiency: A comprehensive review. Radiographics 29, 1909-1920 (2009)

21. Martínez M: Neumopathies in patients with primary immunodeficiencies in treatment with intravenous gammaglobulin. Rev Alerg Mex 54, 14-19 (2007)

22. $\mathrm{Pu} \mathrm{C}$, Sukhal S, Fakhran S: Humoral immunity in bronchiectasis: Finding Good's syndrome. Case Rep Pulmonol 2015, 531731 (2015)

23. Urschel S, Kayikci L, Wintergerst U, Notheis G, Jansson A, Belohradsky BH: Common variable immunodeficiency disorders in children: Delayed diagnosis despite typical clinical presentation. J Pediatr 154, 888-894 (2009)

24. Maglione PJ, Overbey JR, Radigan L, Bagiella E, CunninghamRundles C: Pulmonary radiologic findings in common variable immunodeficiency: Clinical and immunological correlations. Ann Allergy Asthma Immunol 113, 452-459 (2014) 
25. Agondi R, Barros M, Rizzo L, Kalil J, Giavina-Bianchi P: Allergic asthma in patients with common variable immunodeficiency. Allergy $65,510-515$ (2010)

26. Özcan C, Metin A, Erkoçoğlu M, Kocabas CN: Bronchial hyperreactivity in children with antibody deficiencies. Allergol Immunopathol 43, 57-61 (2015)
27. Aghamohammadi A, Allahverdi A, Abolhassani H, Moazzami K, Alizadeh H, Gharagozlou M, Kalantari N, Sajedi V, Shafiei A, Parvaneh N, Mohammadpour M, Karimi N, Sadaghiani MS, Rezaei $\mathrm{N}$ : Comparison of pulmonary diseases in common variable immunodeficiency and X-linked agammaglobulinaemia. Respirology 15, 289-295 (2010) 